\title{
COMPORTAMIENTO MORFOLÓGICO DE CEDRO (Cedrela odorata) Y CAOBA (Swietenia macrophylla) EN RESPUESTA AL TIPO DE SUSTRATO EN VIVERO.
}

\author{
Percy DÍAZ ${ }^{1}$, Denis TORRES ${ }^{2}$, Zylla SANCHEZ ${ }^{1}$, Luis AREVALO' \\ 1 Instituto de Investigaciones de la Amazonía Peruana (IIAP). Programa de Investigación en Manejo Integral del Bosque y \\ Servicios Ambientales (PROBOSQUES).San Martín, Perú. E-mail:pdiaz@iiap.org.pe. \\ 2 Tesista de Pre Grado, Facultad de Ciencias Agrarias de la Universidad Nacional de San Martín - Tarapoto.
}

\section{RESUMEN}

El presente estudio tuvo como objetivo evaluar, en el vivero, el efecto de tres sustratos orgánicos, comparados con el sustrato tradicional, sobre el comportamiento morfológico de plántulas de dos especies, cedro (Cedrela odorata) y caoba (Swietenia macrophylla).

El sustrato tradicional fue tierra agrícola, proveniente de los primeros $10 \mathrm{~cm}$ superficiales. Los sustratos orgánicos fueron una mezcla de $80 \%$ de sustrato tradicional y $20 \%$ de material orgánico: cascarilla de arroz, bagazo de caña y compost de cacao. Las evaluaciones se realizaron a los 90 días luego de repicadas las plántulas. Los resultados indican que el comportamiento morfológico de los Plántulas de caoba y cedro es mejor cuando se utiliza el sustrato con compost de cacao. Los resultados pueden estar relacionados a un mayor aporte de cationes y a la mejora de los parámetros físicos del suelo observados en el sustrato con compost de cacao. El Índice de Calidad de Dickson es mejor para las plántulas de las dos especies producidas en sustratos con compost de cacao comparado con las plántulas crecidas en los otros tres sustratos.

PALABRAS CLAVE: Sustratos, Cedrela odorata, Cedro, Swietenia macrophylla, Caoba.

\section{MORPHOLOGICAL BEHAVIOR CEDAR AND CAOBA IN RESPONSE TO THE TYPE OF SUSTRATE IN TREE NURSERY}

\section{ABSTRACT}

The present study is aimed to evaluate, in nursery, the effect of three organic substrates, compared with the traditional substrate on seedling morphological behavior of two species, cedar (Cedrela odorata) and mahogany (Swietenia macrophylla). The traditional substrate was agricultural land, obtained from the $10 \mathrm{~cm}$. surficial layer of the soil. Organic substrates were a mixture of $80 \%$ of traditional substrate and $20 \%$ organic material: rice husk, bagasse and cocoa compost. Evaluations were performed at 90 days after the seedlings were transplanted to nursery bags. The results indicate that the morphological behavior of cedar and mahogany seedlings were improved when the traditional substrate was mixed with cocoa compost. These results are related to a greater amount of cations and the improvement of physical parameters of soil observed on the substrate with cocoa compost. The Dickson quality index is better for seedlings of the two species produced in the substrate mixed with cocoa compost compared with seedlings produced in the other three substrates.

KEYWORDS: Substrates, Cedrela odorata, Cedar, Swietenia macrophylla, Mahogany. 


\section{INTRODUCCIÓN}

En el vivero, la producción de plántulas de alta calidad requiere de buen material genético y de tecnología adecuada en el proceso de producción. En tal sentido, el sustrato en el que las plántulas desarrollarán sus primeros estadios de vida es fundamental para la obtención de plántulas de calidad (Alorda, 2003).

El sustrato es el medio en el cual las raíces crecerán por un corto periodo de tiempo, siendo su calidad muy importante, ya que de ella depende la calidad inicial de las plántulas (Puerta et al., 2012). El sustrato puede estar compuesto de un solo material o de mezclas.

Los sistemas de producción intensivos y extensivos de arroz, caña de azúcar, cacao y otros, producen anualmente, en la región San Martín, grandes cantidades de residuos orgánicos, tales como la cascarilla de arroz, bagazo de caña de azúcar y cáscara de cacao (GORESAM, 2008). Estos residuos, pueden ser utilizados como sustratos; algunos de ellos deben pasar por un proceso de compostaje previo a su utilización (Cruz et al., 2013).

Por lo general, estos residuos contienen nutrientes que pueden perderse si son quemados o eliminados fuera del campo. En muchos casos, estos residuos son acumulados a la intemperie exponiéndose a un proceso de descomposición, sin ningún tipo de tratamiento y utilización posterior (Basanta et al., 2007). Los residuos orgánicos han sido utilizados con éxito cuando son reciclados a través del compostaje (Carrión et al., 2008). La producción de compost es una forma de utilizar eficientemente estos residuos orgánicos (Laos et al., 2002; Ostos et al., 2008), tanto para ser utilizados como parte del sustrato para la producción de plántulas en el vivero, como para producir abono orgánico destinado a plantaciones de diversas especies agrícolas o agroforestales (Prieto, 2005; De Grazia, et al, 2007). El presnte estudio tuvo como objetivo evaluar, en el vivero, el efecto de tres sustratos orgánicos, comparados con el sustrato tradicional, sobre el comportamiento morfológico de plántulas de cedro y caoba.

\section{MATERIAL Y MÉTODOS}

El ensayo se realizó en el vivero forestal de la SedeSan Martín del Instituto de Investigaciones de la Amazonía peruana, ubicado en el Distrito de Morales, Región San Martín, a $06^{\circ} 28^{\prime} 44^{\prime \prime}$ de Latitud Sur y $76^{\circ} 37^{\prime} 36^{\prime \prime}$ de Longitud Oeste, a 311 msnm.

Se evaluó el comportamiento morfológico de dos especies forestales, Cedro (Cedrela odorata) y
Caoba (Swietenia macrophylla) en respuesta a tres sustratos orgánicos comparados con el sustrato tradicional. El sustrato tradicional (ST) fue suelo agrícola obtenido de los $10 \mathrm{~cm}$. superficiales; los sustratos orgánicos fueron una mezcla de $80 \%$ de ST con $20 \%$ de: cascarilla de arroz (CA), bagazo de caña de azúcar (BCA) o compost de cáscara de cacao (C).

El ensayo fue conducido en parcelas divididas, ubicando las especies forestales Cedro (Cedrela odorata) y Caoba (Swietenia macrophylla) en parcelas principales y los tres tipos diferentes de sustratos orgánicos, comparados con el sustrato tradicional, en sub parcelas. Se instalaron cuatro repeticiones. Para cada unidad experimental, combinación de especie forestal con sustrato, se evaluaron 10 plántulas seleccionadas al azar.

El análisis se realizó utilizando el SAS (Statistical Analysis System, 2004), las fuentes de variación significativamente diferentes se compararon mediante la prueba de Duncan (Gómez y Gómez 1984).

A los 90 días se procedió a medir el diámetro del tallo en milímetros, con un vernier digital a dos centímetros por encima de la línea del sustrato. $\mathrm{La}$ altura de la planta, en centímetros, se midió desde el nivel del sustrato hasta la yema apical, (Pina \& Arboleda, 2010). Las mediciones se iniciaron 10 días después del traslado de las plántulas de las camas de vivero a las bolsas almacigueras.

Para determinar el comportamiento morfológico de las plántulas, se calcularon los siguientes parámetros: incremento de diámetro (ID), expresado en mm. díia ${ }^{-1}$; incremento de altura (IA), expresado en cm.dia ${ }^{-1}$; longitud de raíces secundarias (LR), expresada en $\mathrm{cm} ; \mathrm{n}^{\circ}$ de Raíces, como el número de raíces que fueron medidas. Los incrementos diarios (ID, IA) se calcularon siguiendo el procedimiento descrito por Gardner et al, 2003; Hernández, 1996; usando la relación $\left[\left(\mathrm{t}_{2}-\mathrm{t}_{1}\right) \mathrm{n}\right]$, en donde $t_{2}$ es la medida del parámetro altura o diámetro a los 90 días, $\mathrm{t}_{1}$ medida inicial del parámetro altura $\mathrm{o}$ diámetro, n es igual a 90 días. Para el análisis estadístico del número de raíces, los datos fueron normalizados mediante la fórmula raíz cuadrada $(\sqrt{x}),($ Gómez \& Gómez, 1984).

La calidad de planta fue evaluada en base a los parámetros: peso seco total (PS), expresado en gramos; relación peso seco y peso seco radicular (PSA/PSR); índice de esbeltez (IE), calculado como el cociente entre la altura y el diámetro del tallo; índice de calidad de Dickson (ICD), calculado como cociente entre el peso seco total y la sumatoria de IE y PSA/PSR, (Dickson et al, 1960).

Para determinar el PS, se seleccionaron 10 plantas de cada tratamiento para cada especie y se procedió 
a registrar el peso fresco inicial de la parte foliar y radicular por separado y luego fueron secados en una estufa a $70{ }^{\circ} \mathrm{C}$ por 72 horas, consiguiéndose el peso seco constante de ambas partes de la planta.

Estos resultados sirvieron para obtener la relación PSA/PSR y el índice de calidad de Dickson, siguiendo la metodología de Fonseca et al. (2002), donde los valores más altos indican plantas de mejor calidad (Thompson, 1985).

El manejo de las semillas y plántulas fue homogéneo antes de iniciar el ensayo. Se utilizaron en el estudio semillas sexuales provenientes de árboles "plus" de caoba y cedro que fueron seleccionados en los alrededores de la ciudad de Tarapoto, para ello se realizó la evaluación fenotípica del árbol candidato y la comparación de su superioridad en relación con sus mejores vecinos en un radio inmediato de 10 a 15 $\mathrm{m}$ (Murillo et al, 2010). Las semillas fueron pregerminadas en sustratos de arena fina previamente desinfectada utilizando agua caliente (Camejo \& Añez, 2009), luego se procedió a la siembra en hileras de 1000 semillas por especie, con la finalidad de asegurar el número y calidad de plantas requerido para el repique a las bolsas almacigueras, lo que se realizó diez días después de la germinación.

Para evitar la presencia de hongos, bacterias o insectos en los sustratos estudiados, se desinfectaron con agua caliente a $100^{\circ} \mathrm{C}$ (Camejo \& Añez, 2009), cubriéndose con plástico transparente de $0.4 \mathrm{~mm}$ de espesor por un día. Los sustratos tratados fueron colocados en bolsas de plástico de 250 gramos de capacidad con dimensiones de: $18 \mathrm{~cm}$ x $10 \mathrm{~cm}$ x 0.2 $\mathrm{mm}$. Se tomó una muestra de suelo de cada uno de los sustratos, siendo secada cada una de ellas al medio ambiente, embolsadas, etiquetadas y enviadas al laboratorio de suelos de la Universidad Nacional de San Martín - Tarapoto, para ser analizadas.

Durante el tiempo de ejecución del estudio, las plántulas fueron regadas dos veces al día y el control de plagas se realizó utilizando plaguicidas preventivos con aceptación ambiental, Carbendazim (fungicida) y Clorpirophos (insecticida) para repeler el ataque de hormigas cortadoras de hojas. Las plantas estuvieron en el vivero por 4 meses, diez días entre la germinación y el repique a las bolsas almacigueras y 90 días de duración del ensayo. El vivero estuvo protegido con una malla de $80 \%$ de sombra, para regular la temperatura y el paso de la radiación solar. La humedad relativa en el interior del vivero fue homogénea, con un promedio de $71.6 \%$, siendo la temperatura media de $31.9^{\circ} \mathrm{C}$. Entre las plantas, la humedad relativa fue de $83 \%$ y la temperatura fue en promedio de $30.8^{\circ} \mathrm{C}$.

\section{RESULTADOS Y DISCUSIÓN}

Efecto de los sustratos orgánicos en el comportamiento morfológico de plántulas de C. odorata y S. macrophylla El comportamiento morfológico de la planta es la manifestación fisiológica en respuesta a las condiciones ambientales, al potencial genético y a las prácticas culturales del vivero (Birchler et al., 1998). El análisis de varianza, Tabla 1, indica que existe diferencias significativas entre especies, sustratos y la interacción especies x sustratos para todos los parámetros medidos. El análisis de los parámetros medidos para cada fuente de variación por separado, especies y sustratos, se realizó través de la prueba de rangos múltiples de Duncan al 5\%, Tablas 2 y 3.

La Tabla 1 muestra que el tratamiento compost de cáscara de cacao (C), propició los ID y los IA significativamente mayores para ambas especies, comparado con los otros tratamientos. Los ID para cedro y caoba son 0.052 y 0.050 mm.dia ${ }^{-1}$ respectivamente. Los IA son 0.300 y $0.210 \mathrm{~cm} \cdot \mathrm{dia}^{-1}$ respectivamente para cedro y caoba.

El diámetro al cuello de la planta, es uno de los atributos morfológicos más utilizados en la caracterización de la calidad de planta debido a que permite predecir la supervivencia de la planta en campo (Prieto et al., 2009) y da una aproximación de la sección transversal del transporte de agua y de la resistencia mecánica (Boyer \& South, 1987 citados por Birchler et al., 1998). En consecuencia, para ambas especies, se obtiene mejor calidad de planta con el tratamiento de suelo tradicional enmendado con compost de cáscara de cacao. Birchler et al., (1998), propone no tomar la altura de planta como un buen parámetro, ya que ofrece una ligera aproximación del área fotosintética y de transpiración e ignora la arquitectura del tallo, además si las plántulas son sembradas a alta densidad generan crecimiento por competencia por luz (Negreros et al., 2010); mientras que la tasa de crecimiento (IA) está basada en las fuerzas anabólicas o construcción metabólica como el potencial biótico, actividad fotosintética y absorción nutrimental (Zeide, 1993). En ensayo en los que se utilizó compost de cacao + suelo con plantas de tomate, Lycopersicon esculentum M, (Ibarra, 1997) y soya, Glycine max, en un suelo Ultisol, Atere \& Olayinka (2012) encontraron que el compost de cáscara de cacao mejoró significativamente el crecimiento y el rendimiento.

Con el sustrato CA se obtuvo una mayor longitud de las raíces secundarias en ambas especies. Para cedro, $23.38 \mathrm{~cm}$ y para caoba, $17,75 \mathrm{~cm}$.; $\sin$ embargo, la mayor longitud de las raíces no se reflejó en un mayor ID o IA. Un mayor número de raíces se obtuvo con ST para ambas especies, 13,37 cm. para 
cedro y 13,01 cm. para caoba; estas resultados no se reflejaron en mayores ID o IA. Según Gonzáles (1995), una mayor longitud y número de raíces nos garantizaría mejor desempeño en campo, ya que cuanto más grande sea el sistema radicular de la planta, más puntos de crecimiento se tendrán y habrá una mayor capacidad de explorar el suelo para captar nutrientes. La obtención en vivero de sistemas radiculares más abundantes y mejor desarrollados tiene una estrecha relación con la capacidad absorbente de la planta, por lo tanto, pueden pronosticar una mejor supervivencia en campo definitivo (Oliet, 2000). Por otro lado, hay estudios con cascarilla de arroz, como el desarrollado por López et al. (2008), en diferentes sustratos; suelo:cascarilla de arroz $(1: 1 \mathrm{v} / \mathrm{v})$ y arena; suelo: cascarilla de arroz $(1: 1: 1 \mathrm{v} / \mathrm{v} / \mathrm{v})$ y mantenidos en condiciones de alta humedad relativa $(90 \%)$ y temperatura media de $24{ }^{\circ} \mathrm{C}$. en donde las raíces de uchuva (Physalis peruviana L.) obtuvieron mayor longitud radicular, mayor peso de materia fresca y seca, por lo que se considera que el sustrato facilitó el buen crecimiento radicular, debido a que actuó como un mejorador de las condiciones físicas del sustrato. Las referencias bibliográficas difieren de lo observado en este ensayo, ya que los parámetros mayores de longitud y número de raíces no corresponden con los de ID e IA más altos.

\section{Efecto de los sustratos en la calidad de plántulas de C. odorata y S. macrophylla}

El ANOVA, Tabla 1, para los parámetros de calidad de planta (PS, PSA/PSR, IE, ICD) resulta en especies, sustratos y la interacción especies $\mathrm{x}$ sustratos para todos los parámetros medidos.

La prueba de rangos múltiples de Duncan al 5\%, Tabla 3, muestra que se obtuvieron PS significativamente mayores utilizando el sustrato $C$; 3.28 g. y 3.16 g. para cedro y caoba respectivamente. La producción de biomasa (PS) es importante debido a que refleja el desarrollo de la planta en vivero, sugiriéndonos un alto grado de supervivencia en campo definitivo, mayor capacidad de transformación de la energía en biomasa, debido a una mayor capacidad para realizar procesos fotosintéticos, asegurando un buen crecimiento inicial, estando muy asociado con la altura, diámetro, área foliar, actividad fotosintética y potencial de crecimiento radical (Thompson, 1985; Mexal \& Landis, 1990).

Para cedro se obtuvo una relación PSA/PSR igual para los sustratos BCA y C que fue significativamente más alta que para los sustratos ST y CA. Para esta especie el IE no mostró diferencias significativas para ningún sustrato. Para caoba se obtuvieron resultados más altos de PSA/PSR para los sustratos ST y CA; el IE es significativamente más alto para el sustrato ST y este es significativamente más alto que para ambas especies y para todos los sustratos.

Según Rodríguez (2008), una buena relación PSA/PSR debe fluctuar entre 1.5 y 2.5 , ya que esta proporción PSA con respecto a PSR favorece un proceso fotosintético eficiente y predice resistencia a los periodos relativamente secos (Thompson, 1985). Valores mayores indican desproporción y la existencia de un sistema radical insuficiente para proveer de energía a la parte aérea de la planta. Las mejores relaciones PSA/PSR se obtuvieron con los tratamientos ST y CA para cedro y BCA y C para caoba. Cabe mencionar que tanto Oliet (2000), como Cobas (2001) indican que el factor determinante para la supervivencia de las plantas en campo definitivo es el peso seco radicular, más que el peso seco de la parte aérea, ya que este atributo pronostica mucho mejor la supervivencia. Por otro lado, Mateo et al (2011), indica que en especies de latifoliadas tropicales, la relación PSA/PSR es deseable que sea mayor a 2.0, sobre todo para plantas destinadas a sitios con disponibilidad de agua normal para su tipo de vegetación (selva alta perennifolia, de los 0 a los $750 \mathrm{msnm}$ ), por lo que se considera que los resultados obtenidos con BCA y $\mathrm{C}$ son adecuados para el cedro. Para caoba son mejores los sustratos STy CA.

De acuerdo a Rodríguez (2008), el IE debe ser menor a 6, indicando que se trata de árboles más bajos y gruesos, asociados a una mejor calidad e indican plantas más robustas y con tallos vigorosos, a diferencia de los valores mayores de 6 que indican desproporción con tallos elongados y con diámetros delgados (Prieto et al., 2009). En este ensayo, todos los IE, con excepción de los calculados para los sustratos ST y BCA con caoba, son adecuados.

En relación al Índice de Calidad de Dickson (ICD), combina la información de los dos índices anteriores y los ajusta por el efecto del tamaño de la planta, significando que un mayor índice representa plantas de mejor calidad, lo cual implica que la planta tiene un buen desarrollo y que al mismo tiempo las fracciones aérea y radiculares están equilibradas (Oliet, 2000). Para el presente estudio los mayores ICD se obtuvieron con el sustrato BCA (0.37) y C (0.39) para cedro y con el sustrato $C(0.43)$ para caoba. Los resultados indican que para ambas especies el sustrato $\mathrm{C}$ favorece la producción de plántulas con un equilibrio en la distribución de la masa y la robustez, que evitará seleccionar plantas desproporcionadas y descartar plantas de menor altura pero con mayor vigor (Fonseca et al., 2002).

La mejor calidad de planta se obtiene cuando la parte aérea es relativamente grande y la raíz mediana, lo 
que puede garantizar una mayor supervivencia, ya que evita que la absorción exceda la capacidad de transpiración, dado que el hábitat natural del cedro y la caoba es de alta humedad (Mateo et al., 2011), las plantas producidas en este ensayo serian adecuadas para los lugares en que normalmente se desarrollan estas especies.

\section{Resultados del análisis de las propiedades físico químicas de los sustratos ensayados.}

Las características físicas y químicas de los diferentes tipos de sustratos del ensayo se muestran en la Tabla 4. Las enmiendas con CA, BCA y C causaron el incremento del volumen del suelo, disminución en la densidad aparente y el volumen total de poros. El pH se incrementó con el uso de las tres enmiendas, lo mismo que la Capacidad de Intercambio de Cationes (CIC), ambos debido al incremento de los cationes básicos causado por la adición de las enmiendas. El fósforo y el potasio se incrementaron con todas la enmiendas y el incremento de potasio fue muy notorio con la adición de $\mathrm{C}$.

La adición de $\mathrm{C}$ resultó en altos contenidos de $\mathrm{Ca}$, $\mathrm{Mg}$, K y $\mathrm{P}$, lo que contribuyó a la producción de Plántulas de cedro y caoba con buenas características morfológicas y de calidad. Los altos niveles de elementos catiónicos, como calcio, magnesio y potasio, además de la concentración del fósforo, cuyas funciones son las de promover el crecimiento, mejorar los procesos fotosintéticos, que promueve una mejor conformación de las paredes celulares, se tradujo en una mayor producción de materia seca (Landis, 1989b). Estos datos son similares a los establecidos por Constantino (2011), quien probó la adición combinada de cáscara de cacao al 35\%, más la inoculación de micorrizas del género Glomus intraradices, registrando la mejor interacción sobre el crecimiento y la biomasa de las plántulas de papaya.
El uso de CA redujo la densidad aparente del sustrato, lo que favoreció un mayor crecimiento de la raíz principal, ya que este sustrato proporciona espacios porosos más grandes por su baja densidad. $\mathrm{Su}$ uso puede resultar ventajoso en los viveros forestales. (Landis, 1989a). Steffen et al., (2010) indican que la cascarilla de arroz presenta características favorables para el crecimiento de plantas, siendo considerada un acondicionador de sustrato. Proporciona buena oxigenación para las raíces, drenaje rápido y eficiente, elevado espacio de aeración, relativa estabilidad de estructura, baja densidad y $\mathrm{pH}$ próximo a la neutralidad.

La adición de nutrientes aportados por las enmiendas ensayadas contribuyen al buen crecimiento de las plántulas en vivero (Villar, 2002).

\section{CONCLUSIÓN}

En este ensayo el uso de compost de cacao resultó con un mejor comportamiento morfológico en plantas de cedro y caoba, lo que contribuye a elevar la probabilidad de éxito en la plantación y disminuye el periodo de permanencia en vivero de las plántulas. Este resultado pudo ser causado por la mayor disponibilidad de nutrientes en el suelo enmendado con compost de cáscara de cacao. Los índices de calidad coincidentemente indican que el sustrato con compost de cacao contribuye a la producción de plantas más aptas para ser llevadas a la plantación definitiva para las dos especies ensayadas.

La inclusión de diversos materiales orgánicos al sustrato tradicional, mejoraron drásticamente las propiedades físicas y químicas de los sustratos estudiados, permitiendo una mayor disponibilidad de nutrientes para los Plántulas de las especies forestales probadas.

Los resultados sugieren la posibilidad de obtener plantas de alta calidad, combinando los sustratos compost de cáscara de cacao con cáscara de arroz. 
Tabla 1. Significancias obtenidas en el análisis de variancia para el comportamiento morfológico de plántulas de las especies cedro (Cedrela odorata) y caoba (Swietenia macrophylla) en respuesta a los sustrato ensayados.

\begin{tabular}{|c|c|c|c|c|c|c|}
\hline FV & gl & $\mathrm{CM}$ & $\operatorname{Pr}>F$ & Signif. & $\mathbf{R}^{2}(\%)$ & C.V (\%) \\
\hline \multicolumn{7}{|l|}{ I. D } \\
\hline Especies (E) & 1 & 0.0001671 & 0.0037 & $* *$ & 96.46 & 12.03 \\
\hline Sustratos (S) & 3 & 0.0019333 & $<.0001$ & $* *$ & & \\
\hline$E^{*} S$ & 3 & 0.0004306 & $<.0001$ & $* *$ & & \\
\hline \multicolumn{7}{|l|}{ I.A } \\
\hline Especies & 1 & 0.0482517 & $<.0001$ & $* *$ & 96.92 & 12.51 \\
\hline Sustratos & 3 & 0.0510244 & $<.0001$ & $* *$ & & \\
\hline$E^{*} S$ & 3 & 0.0030027 & 0.0013 & $* *$ & & \\
\hline \multicolumn{7}{|l|}{ L.R } \\
\hline Especies & 1 & 31.007813 & 0.0001 & $* *$ & 90.73 & 6.77 \\
\hline Sustratos & 3 & 31.700521 & $<.0001$ & $* *$ & & \\
\hline$E^{*} S$ & 3 & 30.325521 & $<.0001$ & $* *$ & & \\
\hline \multicolumn{7}{|l|}{ № Raíces } \\
\hline Especies & 1 & 36.872578 & $<.0001$ & $* *$ & 98.18 & 3.91 \\
\hline Sustratos & 3 & 37.266128 & $<.0001$ & $* *$ & & \\
\hline$E^{*} S$ & 3 & 8.108695 & $<.0001$ & $* *$ & & \\
\hline \multicolumn{7}{|l|}{ PST $(g)$} \\
\hline Especies & 1 & 1.1784963 & 0.0008 & $* *$ & 94.97 & 12.31 \\
\hline Sustratos & 3 & 5.7345258 & $<.0001$ & $* *$ & & \\
\hline$E^{*} S$ & 3 & 2.0391448 & 0.001 & $* *$ & & \\
\hline \multicolumn{7}{|l|}{ PSA/PSR } \\
\hline Especies & 1 & 0.0957031 & 0.3644 & ns & 94.35 & 12.21 \\
\hline Sustratos & 3 & 1.1181615 & 0.0004 & $* *$ & & \\
\hline$E^{*} S$ & 3 & 9.6913865 & $<.0001$ & $* *$ & & \\
\hline \multicolumn{7}{|l|}{ I.E } \\
\hline Especies & 1 & 21.043828 & $<.0001$ & $* *$ & 87.12 & 9.69 \\
\hline Sustratos & 3 & 1.4983115 & 0.0059 & $* *$ & & \\
\hline$E^{*} S$ & 3 & 1.1368198 & 0.0173 & * & & \\
\hline \multicolumn{7}{|l|}{ I.C.D } \\
\hline Especies & 1 & 0.00605 & 0.0608 & ns & 93.56 & 14.24 \\
\hline Sustratos & 3 & 0.1023375 & $<.0001$ & $* *$ & & \\
\hline$E^{*} S$ & 3 & 0.024975 & 0.0001 & $* *$ & & \\
\hline
\end{tabular}

${ }^{* *}=$ Significativo con $\mathrm{p}<0.01 ;{ }^{*}=$ significativo con $\mathrm{p}<0.05 ; \mathrm{ns}=$ No significativo con $\mathrm{p}=0.05$ 
Tabla 2. Respuesta de las características morfológicas de Cedro (C. odorata) y Caoba (S. macrophylla) al efecto de cuatro sustratos en vivero $(p<0,05)$.

\begin{tabular}{|c|c|c|c|c|c|c|c|c|}
\hline \multirow{2}{*}{$\begin{array}{l}\text { Parámetros } \\
\text { morfológicos }\end{array}$} & \multicolumn{4}{|c|}{ Cedro (C. odorata) } & \multicolumn{4}{|c|}{ Caoba (S. macrophylla) } \\
\hline & ST & CA & BCA & $\mathbf{C}$ & ST & CA & BCA & $\mathbf{C}$ \\
\hline ID (mm día $\left.a^{-1}\right)$ & $0.030 \mathrm{bc}$ & $0.010 \mathrm{~d}$ & $0.046 \mathrm{a}$ & $0.052 \mathrm{a}$ & $0.011 d$ & $0.025 \mathrm{c}$ & $0.033 \mathrm{~b}$ & $0.050 \mathrm{a}$ \\
\hline IA (cm día $\left.{ }^{-1}\right)$ & $0.180 \mathrm{c}$ & $0.078 \mathrm{e}$ & $0.216 b$ & $0.300 \mathrm{a}$ & $0.068 \mathrm{e}$ & $0.055 \mathrm{e}$ & $0.013 d$ & $0.210 b c$ \\
\hline $\operatorname{LR}(\mathrm{cm})$ & $16.56 \mathrm{~b}$ & $23.38 \mathrm{a}$ & $14.69 \mathrm{c}$ & $15.75 b c$ & $15.25 b c$ & $17.75 \mathrm{bc}$ & $15.75 b c$ & $15.75 b c$ \\
\hline Nº Raíces & $13.37 \mathrm{a}$ & $11.41 \mathrm{~b}$ & $7.03 \mathrm{e}$ & $7.31 \mathrm{e}$ & $13.01 \mathrm{a}$ & $12.95 \mathrm{a}$ & $11.31 \mathrm{c}$ & $10.43 \mathrm{~d}$ \\
\hline
\end{tabular}

${ }^{*}$ Medias con diferentes letras en una misma fila difieren estadísticamente según la Prueba de Duncan $(p<0,05)$.

Tabla 3. Respuesta de la calidad de planta de Cedro (C. odorata) y Caoba (S. macrophylla) al efecto de cuatro sustratos en vivero $(p<0,05)$.

\begin{tabular}{l|c|c|c|c|ccc|c}
\hline \multirow{2}{*}{$\begin{array}{c}\text { Parámetros } \\
\text { de calidad }\end{array}$} & \multicolumn{4}{|c|}{ Cedro (C. odorata) } & \multicolumn{4}{c}{ Caoba (S. macrophylla) } \\
\cline { 2 - 9 } & ST & CA & BCA & C & ST & CA & BCA & C \\
\hline PS Total (g) & $1.46 \mathrm{~d}$ & $0.44 \mathrm{e}$ & $2.82 \mathrm{~b}$ & $3.28 \mathrm{a}$ & $2.10 \mathrm{c}$ & $2.11 \mathrm{c}$ & $2.16 \mathrm{c}$ & $3.16 \mathrm{ab}$ \\
PSA/PSR & $2.12 \mathrm{e}$ & $1.61 \mathrm{f}$ & $3.47 \mathrm{bc}$ & $3.91 \mathrm{ab}$ & $4.22 \mathrm{a}$ & $3.08 \mathrm{~d}$ & $1.52 \mathrm{f}$ & $1.85 \mathrm{ef}$ \\
IE & $4.66 \mathrm{~d}$ & $4.12 \mathrm{~d}$ & $4.17 \mathrm{~d}$ & $4.79 \mathrm{~cd}$ & $7.08 \mathrm{a}$ & $5.63 \mathrm{~b}$ & $6.09 \mathrm{~b}$ & $5.42 \mathrm{bc}$ \\
ICD & $0.22 \mathrm{~d}$ & $0.08 \mathrm{f}$ & $0.37 \mathrm{bc}$ & $0.39 \mathrm{~b}$ & $0.19 \mathrm{e}$ & $0.24 \mathrm{~cd}$ & $0.29 \mathrm{c}$ & $0.43 \mathrm{a}$ \\
\hline
\end{tabular}

*Medias con diferentes letras en una misma fila difieren estadísticamente según la Prueba de Duncan $(p<0,05)$.

Tabla 4. Resultados del análisis de las propiedades físico químicas de los cuatro sustratos ensayados.

\begin{tabular}{|c|c|c|c|c|c|}
\hline \multirow{2}{*}{ Propiedades } & \multirow{2}{*}{ Unidades } & \multicolumn{4}{|c|}{ Sustratos } \\
\hline & & ST & CA & BCA & C \\
\hline \multirow{4}{*}{ Físicas } & Volumen $\left(\mathrm{cm}^{3}\right)$ & 287.2 & 364.5 & 299.5 & 292.5 \\
\hline & Densidad aparente $\left(\mathrm{g} \mathrm{cm}^{3}\right)$ & 1.27 & 0.46 & 0.97 & 1.00 \\
\hline & Vol. Total Poros $\left(\mathrm{g} \mathrm{cm}^{3}\right)$ & 78.75 & 50.00 & 68.75 & 59.00 \\
\hline & $\%$ Humedad & 16.28 & 20.96 & 18.63 & 19.60 \\
\hline \multirow{11}{*}{ Químicas } & $\mathrm{pH}$ & 5.51 & 7.44 & 7.17 & 7.46 \\
\hline & C.E. $\left(d S m^{-1}\right)$ & 0.106 & 0.010 & 0.788 & 0.900 \\
\hline & $\% \mathrm{M} .0$ & 3.78 & 6.65 & 4.76 & 6.65 \\
\hline & $\% \mathrm{~N}$ & 0.19 & 0.33 & 0.24 & 0.33 \\
\hline & $\mathrm{P}(\mathrm{ppm}) \mathrm{mg} \mathrm{kg}^{-1}$ & 9 & 13 & 12 & 15 \\
\hline & $\mathrm{K}(\mathrm{ppm}) \mathrm{mg} \mathrm{kg}^{-1}$ & 38.56 & 621.9 & 304 & 368.4 \\
\hline & C.I.C & 2.71 & 15.03 & 11.27 & 15.27 \\
\hline & $\mathrm{Ca}^{++} \mathrm{cmol} \mathrm{kg}^{-1}$ & 2.08 & 4.44 & 5.45 & 7.61 \\
\hline & $\mathrm{Mg}^{++} \mathrm{cmol} \mathrm{kg}^{-1}$ & 0.45 & 8.95 & 4.99 & 6.62 \\
\hline & $\mathrm{Na}^{+} \mathrm{cmol} \mathrm{kg}{ }^{-1}$ & 0.08 & 0.05 & 0.03 & 0.10 \\
\hline & $\mathrm{K}^{+} \mathrm{cmol} \mathrm{kg}^{-1}$ & 0.10 & 1.59 & 0.78 & 0.94 \\
\hline
\end{tabular}

Fuente: Laboratorio de suelos de la Universidad Nacional de San Martín (UNSM-T), 2012. 


\section{BIBLIOGRAFÍA CITADA}

Alorda, M.D. 2003. Estudio de los sustratos utilizados en la producción de plantines forestales en el noreste de Entre Ríos. Trabajo Final de Graduación, Facultad de Ciencias Agropecuarias UNER. 37p.

Atere, C.; Olayinka, A. 2012. Effects of cacao-pod compost with and without starter $\mathrm{N}$ and $\mathrm{P}$ on the growth and yield of soybean in an ultisol. Nigerian Journal of Technological Research $\mathrm{V}$ o $1 \mathrm{u} \mathrm{m}$ e $\mathrm{n} \quad 7, \quad \mathrm{~N}$ o 3 . (http://www.ajol.info/index.php/njtr/article/vie w/88840). Acceso: 21/12/2013.

Basanta, R.; García, M.A.; Cervantes, J.E.; Mata, H.; Bustos, G. 2007. Sostenibilidad del Reciclaje de Residuos de la Agroindustria Azucarera: Una Revisión. Ciencia y Tecnología Alimentaria. Sociedad Mexicana de Nutrición y Tecnología de Alimentos, México. Vol. 5, núm. 4, pp. 293305.

Birchler, T.; Rose R.W.; Royo A.; Pardos M. 1998. La planta ideal: revisión del concepto, parámetros definitorios e implementación práctica. Oregon State University, Oregon. EE.UU y Universidad Politécnica de Madrid, E s p a ̃̃ a . $\quad 13 \quad$ p . I n : (http://www.inia.es/gcontrec/pub/11.T.BIRCH LER_1047630290178.pdf). Acceso: 15/12/2012.

Carrión, C.; García, R.; Fornes, F.; Puchades, R.; Abad, M. 2008. Acidifying compost from vegetable crop wastesto prepare growing media for containerized crops. Compost Sci. Util. 16, 20-29.

Camejo, A.; Añez, M. 2009. Crecimiento de lechosa (Carica papaya L.) cv. "Maradol" en dos tipos de envase y de sustrato. Programa de Ciencia del Agro y del Mar. Universidad Nacional Experimental de los Llanos Occidentales "Ezequiel Zamora" (UNELLEZ). Guanare. Estado Portuguesa. Venezuela. Revista UDO Agrícola 9(1): 136-140.

Cobas, M. 2001. Caracterización de los atributos de la calidad de la planta Hibiscus elatus cultivada en tubetes. Tesis presentada en opción al grado Científico de Doctor en Ciencias Forestales. Facultad de Agronomía y Forestal. Departamento de producción Forestal. UPR. Pinar del Río.

Constantino, M; Gómez, R; Álvarez, J; Pat, J; Espín, E. 2011. Efecto de la inoculación de Azotobacter chroococcum y Glomus intraradices en el crecimiento y nutrición de plántulas de papaya en fase de vivero. Agron. Costarricense [online]. 2011, vol.35, n.1, pp. 15-31. (http://www.scielo.sa.cr/scielo.php?pid=S0377 $-94242011000100002 \&$ script $=$ sci_arttext). Acceso: 20/12/2013.
Cruz, E; Can, A; Sandoval, M.; Bugarín, M.R; Robles, A.; Juárez, L.P. 2012. Sustratos en la Horticultura. Revista Biociencias, V.2(2): 172 6

(http://biociencias.uan.edu.mx/publicaciones/0 3-02/biociencias3-2-2.pdf). Acceso: $10 / 01 / 2014$.

De Grazia, J.; Tittonell, P.A.; Chiesa, A. 2007. Efecto de sustratos con compost y fertilización nitrogenada sobre la fotosíntesis, precocidad y rendimiento de pimiento (Capsicum annuurn). Ciencia e Investigación Agraria, 34(3), 1952

$$
0
$$$$
4
$$

(http://www.scielo.cl/scielo.php?script=sci_artt $\mathrm{e} \quad \mathrm{x} \quad \mathrm{t} \quad \mathrm{p}$ i $\mathrm{d}=\mathrm{S} \quad 0 \quad 7 \quad 1 \quad \overline{8}$ $16202007000300003 \& \operatorname{lng}=\mathrm{es} \& \mathrm{t} \operatorname{lng}=\mathrm{es}$. 10.4067/S0718-16202007000300003.). Acceso: 23/10/2013

Dickson, A.; Leaf, A.L.; Hosnerm J.F.; 1960. Quality appraisal of white spruce and white pine seedling stock in nurseries. For. Chron., 36: 1013.

Fonseca, E.S.; Valeri, E.; Miglioranza, N.; Fonseca L.; Couto, L. 2002. Padrao de qualidade de mudas de Trema micrantha (L.) Blume, producidas sobre diferentes períodos de sombreamento. R. Arvore 26(4): 515-523.

Gardner, F.P.; Pearce R.B.; Mitchell, R.L. 2003. Physiology of crop plants. Blackwell publishing company. Iowa, $326 \mathrm{pp}$.

GORESAM, 2008. Plan Estratégico Sectorial Regional Agrario 2009-2015. Gobierno Regional de San Martín. Dirección Regional de Agricultura de San Martin. Documento de $\begin{array}{llllllllllllll} & \mathrm{r} & \mathrm{a} & \mathrm{n} & \mathrm{s} & \mathrm{p} & \mathrm{a} & \mathrm{r} & \mathrm{e} & \mathrm{n} & \mathrm{c} & \mathrm{i} & \mathrm{a}\end{array}$ (http://www.regionsanmartin.gob.pe/administr acion/documentos_transparencia/doc_transpar encia13.pdf). Acceso: 26/11/2013

Gómez, A.K.; Gómez, A.A. 1984. Statistical Procedures for Agricultural Research. 2da. Ed. John Willey y Sons Inc. USA. 680p.

Gonzáles, K.V. 1995. Tipos de envases en viveros forestales. In: Viveros forestales. Publicación especial No. 3. Centro de Investigación Disciplinaria en Conservación y Mejoramiento de Ecosistemas Forestales. INIFAP SAGARPA. México, D. F. pp. 26-36.

Hernández, G. 1996. Tasa de elongación de frondos de Microcystis pyrifera (L.) A.G. en baja california sur Mexico, Ciencias Marinas, vol. 22, num. 1, pp 57-72.

Ibarra, L. 1997. Efecto de tres sustratos orgánicos y una solución nutritiva en la producción de plántulas de tomate (Lycopersicon esculentum W.). Tesis de maestria en ciencias en suelos. Universidad Autónoma Agraria Antonio Narro, Buenavista, Saltillo, Coahuila (México). 103 p. (ht t p ://orton.catie.ac.cr/cgi- 
bin/wxis.exe/?IsisScript=CENTA.xis\&method $=$ post $\&$ formato $=2 \&$ cantidad $=1 \&$ expresion $=m f$ $\mathrm{n}=000316$ ). Acceso: 02/01/2014.

Landis, T.D. 1989a. Manual de viveros para la producción de especies forestales en contenedor. Volumen dos: contenedores y medios de crecimiento. Departamento de agricultura, servicio forestal. p. 89.

Landis, T.D. 1989b. Manual de viveros para la producción de especies forestales en contenedor. Volumen cuatro: Fertilización y Riego.p. 1-67

Laos, F.; Mazzarino M.J.; Walter, I. Roselli, L; Satti, P.; Moyano, S. 2002. Composting of fish offal and biosolids in northwestern Patagonia. Bioresour. Technol. 81: 179-186.

López, F.; Guio, N.; Fischer, G.; Miranda, D. 2008. Propagación de uchuva (Physalis peruviana L.) mediante diferentes tipos de esquejes y sustratos. Rev.Fac.Nal.Agr.Medellín [online]. 2008, vol.61, n.1 [cited 2014-01-26], pp. 4347$\begin{array}{llll}4 & 3 & 5 & 7\end{array}$. (http://www.scielo.org.co/scielo.php?script=sci a r t t e x t \& p i d $=\mathrm{S} 0304$ $\overline{2} 8472008000100011 \& \operatorname{lng}=\mathrm{en} \& \mathrm{nrm}=\mathrm{iso})$. Acceso: 20/12/2013.

Mateo, J; Bonifacio, R; Pérez, S; Mohedano, L; Capulin, J. 2011. Producción de (Cederela odorata L.), en sustrato a base de aserrín crudo en sistema tecnificado en Tecpan de Galeana, Guerrero, Mexico. Ra Ximhai, Vol. 7, núm. 1. En e r o-a b r i 1. P p $123-132$. ( http://www.redalyc.org/pdf/461/46116742012. pdf). Acceso: 30/11/2013.

Mexal, J.G.; Landis T.D. 1990. Target seedling concepts: height and diameter. In: Target seedlings symposium. Gen. Tech. Rep. USDA Forests. 13:105-119.

Murillo, O.; Picado, F.; Badilla, Y.; Vallejos, J. 2010. "Metodología para la selección e incorporación de árboles plus en programas de mejoramiento genético forestales". Escuela de ingeniería Forestal. Instituto Tecnológico de Costa Rica, Cartago, Costa rica. BARCA S.A.p 45.

Negreros, P.; Apodaca, M.; Mize, C.W. 2010. Efecto de sustrato y densidad en la calidad de plántulas de cedro, caoba y roble. Madera y bosques, $16(2)$ ) $7-18$. (http://www.scielo.org.mx/scielo.php?script=sc i a r t t e x t \& p i d = S 1405 0 $\overline{47} 12010000200001 \& \operatorname{lng}=\mathrm{es} \& \mathrm{t} \operatorname{lng}=\mathrm{es}$. Acceso: 27/11/2013

Oliet, J. 2000. La calidad de la postura forestal en vivero. Escuela Técnica Superior de Ingenieros Agrónomos y de Montes de Córdoba. España. $93 \mathrm{p}$.

Ostos, J.C.; López, R.; Murillo, J.; López, R. 2008. Substitution of peat for municipal solid waste- and sewage sludge-based compost in nursery growing media: Effects on growth and nutrition of the native shrub Pistacia lentiscus L. Bioresour. Technol. 99: 1793-1800.

Pina, M.; Arboleda, M. 2010. Efecto de dos ambientes lumínicos en el crecimiento inicial y calidad de plantas de Crescentia cujete. Bioagro [online]. vol.22, n.1, pp. 61-66. ISSN 13163361.

Prieto, E.S. 2005. Calidad del plantín de tomate (Lycopersicon esculentum Mili.) cultivado en sustratos adicionados con lombricompuesto. Tesis de grado. Facultad de Agronomía, Universidad de Buenos Aires, Buenos Aires, Argentina. 14 pp.

Prieto, R.J.A.; García, R.J.L.; Mejía, B.J.M.; Huchín, A.S.; Aguilar V.J.L. 2009. Producción de planta del género Pinus en vivero en clima templado frío. Publicación Especial Núm. 28. Campo Experimental Valle del Guadiana INIFAP-SAGARPA. Durango, Dgo. México. $48 \mathrm{p}$.

Puerta, C.E.; Russian, T.L.; Ruiz, C.A. 2012. Producción de plántulas de pimentón (Capsicum annuит L.) en sustratos orgánicos a base de mezclas con fibra de coco. UDO Agrícola 12(2):298-306.

Rodríguez, T.D.A. 2008. Indicadores de calidad de planta forestal. Universidad Autónoma Chapingo. Mundi Prensa México. $156 \mathrm{p}$.

SAS Institute. 2004. Statistical Analysis System (Version 9). Copyright (c) by SAS Institute Inc., Cary, NC, USA.

Steffen, P; Antoniolli, Z; Steffen, R; Machado, R. 2010. Casca de arroz e esterco bovino como substratos para a multiplicação de minhocas e produção de mudas de tomate e alface. Acta Zool. Mex [online]. 2010, vol.26, n.spe2, pp. $\begin{array}{llllllll}3 & 3 & 3 & - & 3 & 4 & 3\end{array}$. (http://www.scielo.org.mx/scielo.php?script=sc i $-\mathrm{p}$ d f \& $\mathrm{p}$ i d $=\mathrm{S} \quad 0 \quad 0 \quad 6 \quad 5$ 173̄72010000500025\&lng=es\&nrm=iso\&tlng $=\mathrm{pt}$ ). Acceso: 28/12/2014.

Thompson, B. 1985. Seedling morphological evaluation. What can you tell by looking. In: Evaluating seedling quality: principles, procedures and predictive abilities of major test. M. L. Durges. Forest Research Laboratory. Oregon State University. p. 59-65.

Villar, P. 2002. Importancia de la calidad de planta en las labores de revegetación Resúmenes de ponencias del simposio de la AEET: Restauración de ecosistemas en ambientes mediterráneos. Posibilidades y limitaciones. Ecosistemas 1: 6-7p

Zeide, B. 1993. Analysis of growth equations. Forest Science, vol. 39, no. 3, p. 594-616. 
